\title{
Struma ovarii
}

\author{
Muhammed A. Hatem, Benny So \\ Foothills Medical Center, Calgary, AB, Canada
}

Correspondence: Muhammed A. Hatem. Address: Foothills Medical Center, Calgary, AB, 1403 - 29 Street N.W., Canada. E-mail: asl7mak@gmail.com

Received: September 22, 2014

Accepted: October 16, 2014

Online Published: October 27, 2014

DOI : $10.5430 /$ jbgc.v4n4p22

URL: http://dx.doi.org/10.5430/jbgc.v4n4p22

\section{Abstract}

Struma ovarii is a rare ovarian tumor, most commonly affect patients in their 4th to 6th decade of life, it is benign in the vast majority of cases with excellent prognosis following surgical removal; however, it is malignant in about $5 \%$ of cases with possibility of distant metastasis, radiologically it is difficult to differentiate it from other benign or malignant ovarian tumors.

\section{Key words}

Ovary, Struma, Teratoma, Thyroid, Malignant

\section{Introduction}

Struma ovarii is a rare ovarian tumor, first discovered by Von Kalden in $1895^{[1]}$. It is a form of ovarian teratoma in which thyroid tissue composes more than $50 \%$ of the tumor ${ }^{[2]}$. This tumor is benign in most patients, however, it can be malignant in $5 \%$ of cases ${ }^{[3]}$. Patients with this condition present with pelvic mass with occasional ascitis, since these findings are worrisome for ovarian cancer, these patients usually end up with surgery to remove the tumor ${ }^{[2]}$. Similar to other ovarian tumors, struma ovarii may be solid or cystic, and if it is cystic, it can be unilocular or multilocular ${ }^{[1]}$. In this case report we present a rare case of malignant strauma ovarii presented with wide spread metastasis.

\section{Case report}

A 62 year old female presented with chest pain and cough, plain chest radiograph(see Figure 1) showed multiple bilateral pulmonary nodules. Enhanced chest abdomen and pelvic CT (see Figure 2) demonstrated a big left lower quadrant mass in addition to the pulmonary nodules. Pelvic ultrasonography (see Figure 3) demonstrated the pelvic mass has cystic and solid components with internal vascularity. Percutaneous CT guided biopsy of the solid portion of the mass was performed (see Figure 4). Pathology report identified normal thyroid tissue and favored the diagnosis of struma ovarii.

Whole body iodine study using I-123 (396 MBg) with SPECT CT (see Figure 5 and 6) shows wide spread metastasis of the tumor to the lungs and pelvis. This patient was treated with surgical removal of the primary tumor and adjuvant radioactive Iodine-131 for metastasis. SPECT CT of the chest, abdomen and pelvis using $370 \mathrm{MBq}$ I-123 (see Figure 7) done one year later show significant improvement after treatment. 
Figure 1. Plain chest radiograph posterior anterior and lateral of 62 year old female who presented with chest pain and cough shows bilateral pulmonary nodules with the largest nodule located in the left lower lung.

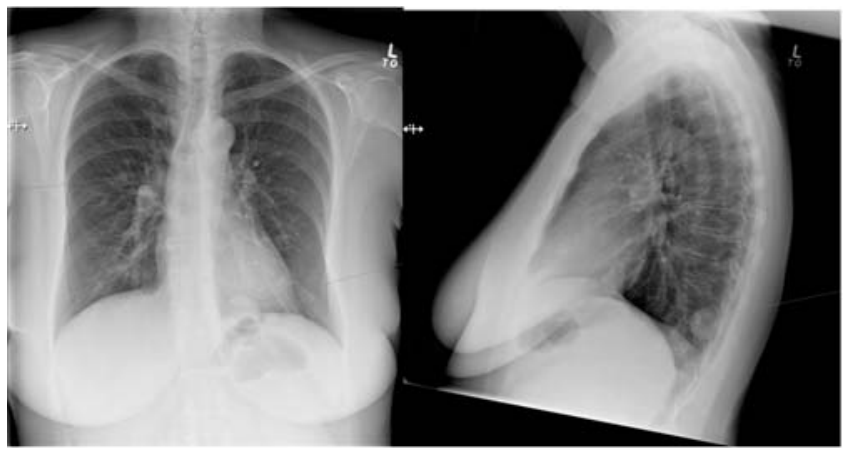

Figure 2. Axial enhanced CT scan image of 62 year old female shows heterogeneously enhancing mass in the left lower quadrant.

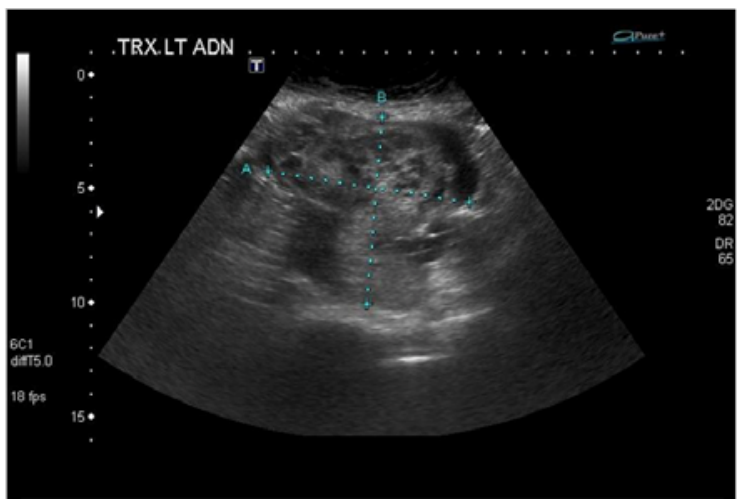

Figure 3a. Transverse left adenexal ultrasound of 62 year old female shows heterogeneous mass with solid and cystic components.

Figure 4. Axial CT scan image of a 62 year old female with big left lower quadrant mass shows the needle tip in the mass during percutaneus CT guided Biopsy.
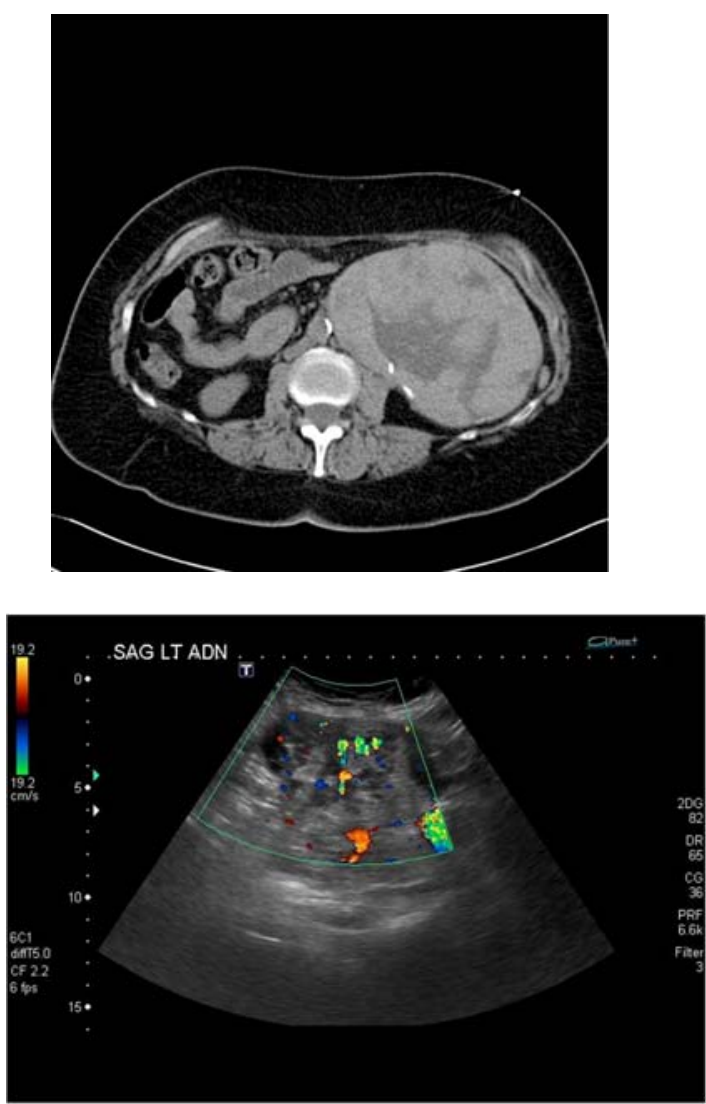

Figure 3b. Sagital left adenexal ultrasound with color Doppler of 62 year old female shows heterogeneous mass with solid and cystic components and some vascularity.

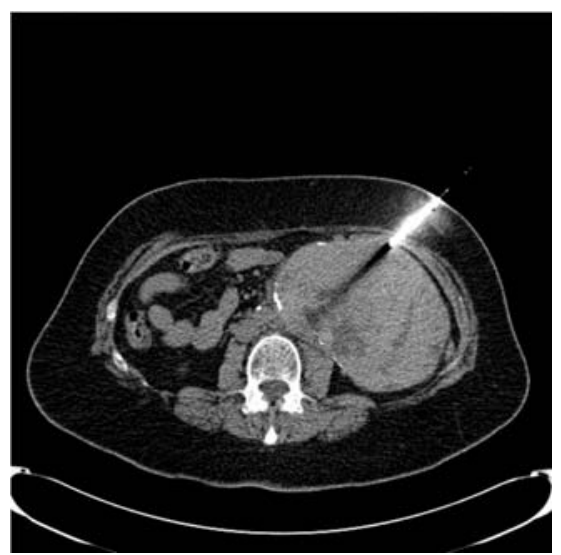


Figure 5. Nuclear Medicine CT/SPECT scan of 62 year old female was done two months after her presentation with multiple pulmonary nodules shows metastasis of the tumor to the pelvis.

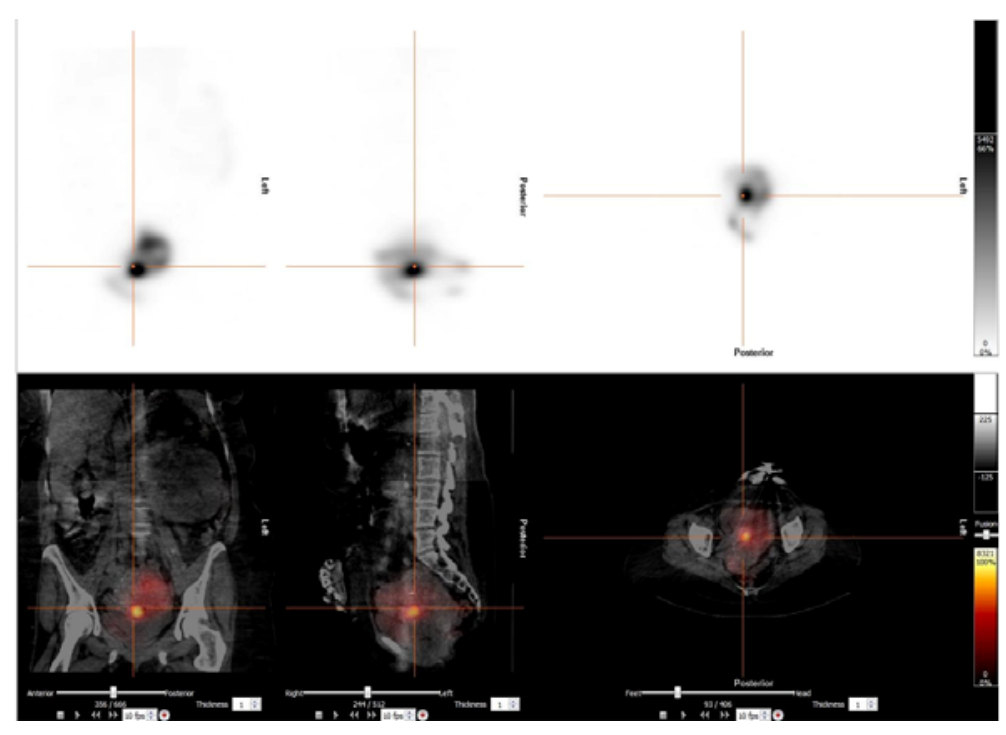

Figure 6. Whole body iodine study using I-123 (396 MBg) with SPECT CT, shows wide spread metastasis of the disease in the lungs and pelvis.

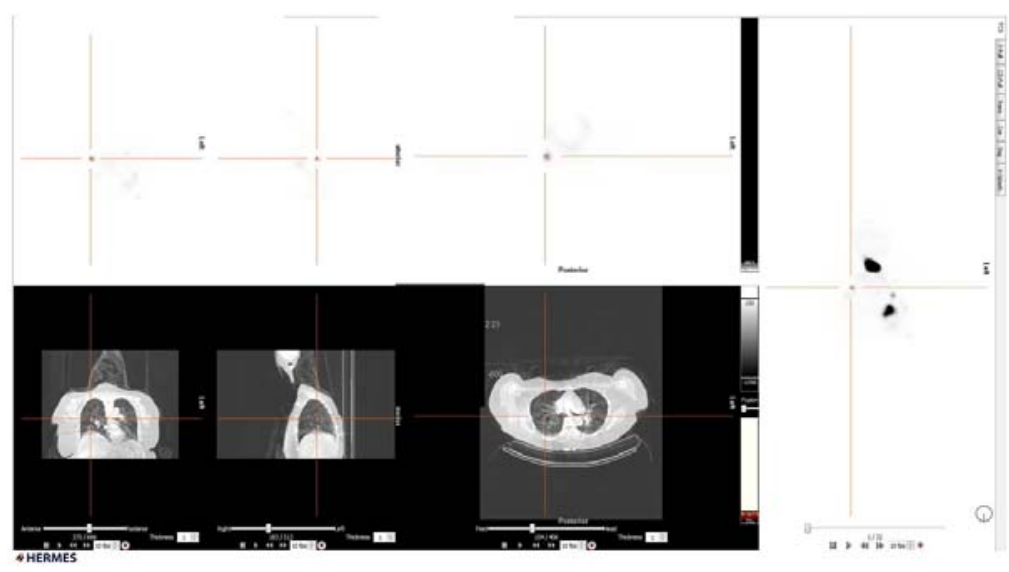

Figure 7. SPECT CT of the chest, abdomen and pelvis using $370 \mathrm{MBq} \mathrm{I}-123$ done one year later show significant improvement in this patient.

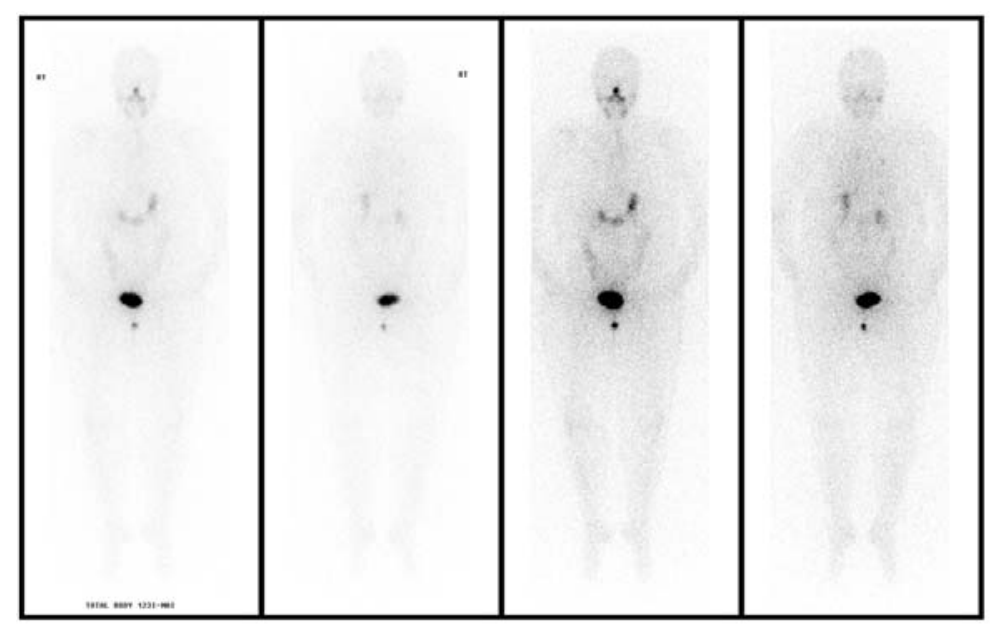

\section{Discussion}

Struma ovarii is a rare tumor, it is a form of ovarian teratoma which is mostly composed of thyroid tissue; this tumor is asymptomatic in majority of cases ${ }^{[4]}$. However, in $5 \%$ of cases it can be symptomatic. Local symptoms can be pelvic pain or discomfort. It can also produce systemic symptoms of thyrotoxicosis ${ }^{[5]}$. This tumor is very rare and makes up only 
$0.3 \%-1 \%$ of all ovarian tumors ${ }^{[1]}$. It is benign in about $95 \%$ of patients. Only small percentage of patients has a malignant course with potential for distant metastasis ${ }^{[6]}$. The prognosis is usually excellent ${ }^{[5]}$.

The peak incidence is between 4th and 6th decades but it can occur at any age group. The current treatment for struma ovarii is surgical resection; however, more extensive research is needed to establish the optimal way of treatment and the decision between surgical resection versus watchful waiting ${ }^{[3]}$.

Imaging findings are nonspecific and cannot easily differentiate struma ovarii from other forms of ovarian tumors.

On CT scan and ultrasound, the lesion appears as a complex adnexial mass with multiple solid and cystic areas, ascitis may be found in a significant number of cases.

On MRI, it may appear as a multiloculated cystic mass with solid components with high or low signal intensity on T1 and T2. Very low signal intensity on T2 is sometimes considered suggestive of struma ovarii due to viscous colloid content of the mass. The solid portion may show enhancement on $\mathrm{T} 1$ with gadolinium contrast administration ${ }^{[7]}$.

Struma ovarii has a wide differential diagnosis as the imaging appearance is non-specific. Depending on size of the presenting lesion and symptoms at presentation, the differential may have to include some or all of the following: benign and malignant ovarian tumors, hydrosalpinx, endometrioma, ectopic pregnancy, tubo ovarian abscess and physiological ovarian cyst in addition to metastatic cancer to ovaries ${ }^{[8]}$. It is worth remembering struma ovarii in the differential diagnosis of a complex adnexal mass.

\section{Conclusion}

Struma ovarii is a rare ovarian tumor that can present with wide spread metastasis, this tumor respond very well to surgical resection of primary tumor and adjuvant radioactive iodine therapy for the metastasis.

\section{References}

[1] K. Van de Moortele, D. Vanbeckevoort, S. Hendrickxl. Struma ovarii: us and CT findings. JBR-BTR. 2003; 86: 209-210. PMid: 14527060 .

[2] Leila Yassa, Peter Sadow, Ellen Marqusee. Malignant struma ovarii. Nature Clinical Practice Endocrinology \& Metabolism. 2008; 4: 469-472. http://dx.doi.org/10.1038/ncpendmet0887

[3] Khediri Z, Mbarki C, Abdelaziz AB, Hsayoui N, Mezghenni S, Oueslati H. Struma ovarii: clinical presentations of an uncommon tumor. International Journal of Case Reports and Images. 2012; 3(4): 10-14. http://dx.doi.org/10.5348/ijcri-2012-04-106-CS-3

[4] M Matsuki, Y Kaji, M Matsuo, Y Kobashi. Struma ovarii: MRI findings. British Journal of Radiology. 2000; 73: 87-90. PMid: 10721328. http://dx.doi.org/10.1259/bjr.73.865.10721328

[5] Christopher P. Wagner, BS, Brandi N. Hicks, MD, Kevin M. Nakamura, MD. Incidental scintigraphic detection of struma ovarii following total thyroidectomy for papillary thyroid cancer. Radiology Case Reports. 2011; 6(3).

[6] Susan J. Ackerman, MD, Lisa F. Baron, MD, Tara C. Noone, MD. Struma ovarii. Applied radiology. April 2002; 31(4).

[7] Dr Frank Gaillard, Dr Yuranga Weerakkody, et al. Struma ovarii tumour. Available from: http://radiopaedia.org/articles/struma-ovarii-tumour. Accessed November/22/2013.

[8] Jeannie Chen Kelly, MD. Struma Ovarii Differential Diagnoses. Available from: http://emedicine.medscape.com/article/256937-differential. Accessed November/22/2013 